\title{
Reconstrucción microquirúrgica del nervio lingual
}

\author{
Claudio Ignacio Huentequeo ${ }^{1,2}$, Saul Siso-Cárdenas ${ }^{1,2}$, \\ Cristopher Mayer-Olivares ${ }^{3,4}$ y Sergio Olate-Morales ${ }^{1,2}$
}

Servicio de Cirugía Oral y Maxilofacial, Hospital de Lautaro, Lautaro, Region de la

Araucanía.

2Servicio de Cirugía

Maxilofacial y de Cabeza y

Cuello, Hospital Complejo

Asistencial Padre Las Casas,

Region de la Araucanía.

3Servicio de Cirugía Oral y

Maxilofacial Hospital Luis Tisne

Brousse, Santiago.

${ }^{4}$ Servicio de Cirugía Oral y

Maxilofacial Hospital De

Salvador, Santiago,

Chile.

Recibido 2020-10-11 y aceptado 2020-12-16

Correspondencia a:

Dr. Claudio Ignacio Huentequeo

claudiohuentequeo@gmail.

com

\section{Microsurgical lingual nerve repair}

Introduction: Lingual nerve injury after a traumatic event is frequent during some maxillofacial procedures, being the third molar extraction the most frequent cause. Lingual nerve injury may be performed in different grades of damage and it is often invalidating. Microsurgical reconstruction is an efficacy technique with a rate of success over $80 \%$. Aim: To present two cases of lingual nerve microsurgical reconstruction after lingual nerve injury. Clinical Cases: Two female patients suffered lingual nerve injury after third molar extraction, both were submitted to lingual nerve reconstruction. It was performed the microsurgery reconstruction of the lingual nerve, both present favorable outcomes follow up to 1.5 years, including positive Functional sensory recovery (FSR +) and sensorial test S3 and S4+ respectively for each patient. Discussion: The timing of lingual nerve microsurgery is not well defined; however, most authors suggest an early surgical treatment before 6 months. The lingual nerve injury often affect the quality of life of the patient. Microsurgery reconstruction should be incorporated into the treatment algorithm of lingual nerve injury.

Key words: microsurgery; lingual nerve; lingual nerve injuries; reconstructive surgical procedures.

\section{Resumen}

Introducción: El daño del nervio lingual posterior a un evento traumático es frecuente durante algunos procedimientos en cirugía maxilofacial. Siendo la desinclusión de terceros molares la causa más frecuente. La reconstrucción microquirúrgica del nervio es una técnica eficaz con éxito sobre el $80 \%$ de los casos. Objetivo: El objetivo de este artículo es presentar dos casos de reconstrucción microquirúrgica del nervio lingual. Casos Clínicos: Pacientes de sexo femenino tratadas por el equipo de Cirugía Maxilofacial del Hospital Dr. Abraham Godoy Peña. Donde se les realiza la reconstrucción microquirúrgica del nervio lingual, ambas presentan resultados positivos al año y medio, con una recuperación funcional sensorial $($ FSR +$)$ y sensorial positiva S3 y S4+ respectivamente para cada paciente. Discusión: El momento de la reconstrucción microquirúrgica del nervio lingual no está bien definido. Sin embargo, la mayoría de los autores sugieren un tratamiento quirúrgico temprano, antes de los 6 meses. El daño del nervio lingual a menudo afecta la calidad de vida del paciente. La reconstrucción microquirúrgica del nervio lingual debe ser incorporada dentro del algoritmo de tratamiento del daño del nervio lingual.

Palabras clave: microcirugía; nervio lingual; daño del nervio lingual; procedimientos quirúrgicos reconstructivos.

\section{Introducción}

El nervio lingual (NL) es rama del nervio trigémino, el cual además recibe fibras provenientes del VII par craneano, con un calibre de aproximadamente entre 1,6 y $3 \mathrm{~mm}$ de diámetro ${ }^{1}$. Debido a su recorrido se encuentra expuesto a lesiones de distintos procedimientos en cirugía maxilofacial: osteotomías, laringoscopías, fracturas, tratamiento de tumores y cáncer, y desinclusiones de terceros molares, siendo este último el procedimiento más prevalente $(0,1 \%$ a un $22 \%)$ y estudiado ${ }^{2-4}$.

Las secuelas de la lesión del NL van a depender del daño, sin embargo, por lo general resulta en un gran impacto en la calidad de vida del paciente, donde un número considerable son irreversibles ${ }^{3}$. Se propone esperar su evolución entre 3 y 6 meses antes de pensar en la cirugía ${ }^{3}$. Estas secuelas van desde hipoestesia o parestesia de los 2/3 anteriores de la lengua, pérdida del gusto, alodinia, hasta do- 
lores neuropáticos ${ }^{3,4}$. Pogrel propone el tratamiento quirúrgico del NL antes de los 3 meses $^{5}$, el año 2007 Susarla et al., proponen un algoritmo de tratamiento para evaluar el tratamiento de los 6 meses, donde la reconstrucción microquirúrgica presenta resultados eficaces sobre el $80 \%$ de éxito en los pacientes ${ }^{6}$. Más tarde, otros autores como Bagheri y Biglioli, muestran que el tratamiento temprano mejora de forma sustancial el éxito de esta cirugía ${ }^{7,8}$.

El tratamiento microquirúrgico de la lesión del NL tiene resultados exitosos ${ }^{9,10}$, siendo la primera elección en pacientes que presentan una parestesia total, alodinia o disestesias ${ }^{11}$, sin embargo, esta cirugía no es frecuentemente utilizada en Latinoamérica. Este artículo tiene como objetivo presentar 2 casos de reconstrucción microquirúrgica del nervio lingual con seguimiento a 18 meses.

\section{Caso 1}

Paciente de sexo femenino, 28 años, acude al Hospital Dr. Abraham Godoy Peña de Lautaro, IX Región; al realizar desinclusión de diente 3.8 se daña la pared lingual. A las $24 \mathrm{~h}$ paciente presenta parestesia lingual (S0) y con pérdida del gusto lado izquierdo. Se procede a controlar semanalmente: función, tacto, dolor, sensibilidad a los cambios de temperatura y gusto. A la sexta semana la paciente presentaba un dolor persistente en la zona de la base de la lengua, además se mantenía la parestesia del lado izquierdo de la lengua, el piso y la encía del mismo lado (S0). Se decide realizar la reconstrucción del nervio lingual (NL). Bajo anestesia general, se realiza el abordaje en el piso de boca en la zona retromolar y extendiéndose desde el segundo molar hasta la rama, a través de disección roma se reconoce el conducto submandibular y el loop del nervio, se realiza la búsqueda de la lesión con el apoyo de microscopía: donde se aprecia el nervio dañado y formando un neuroma (Figura 1). Se procede a realizar la neurólisis y la escisión del neuroma (Figura 2) y se evalúa tensión para luego realizar la reconstrucción del nervio a través de la neurorrafia sobre el epineuro con microcirugía, se utilizan tres puntos de sutura en $360^{\circ}$ con nylon $8 / 0$, se cierra el abordaje con vycril 3/0. A la semana la paciente refería mayor percepción a la presión, sobre todo en la zona más posterior sobre el dorso y la zona lateral (S2). Al mes, además, la paciente siente dolor espontáneo en la punta de la lengua y sensación de electricidad, comienza con dolor quemante e hiperestesia en zona lingual de incisivos inferiores al roce con alimentos $\mathrm{o}$ al tacto. $\mathrm{Al}$ año, paciente sin dolor con $\mathrm{FSR}+$, mejora notablemente el gusto, recupera $100 \%$ el gusto con pérdida de sensibilidad en algunas partes (S3).

\section{Caso 2}

Paciente sexo femenino de 21 años; acude al Hospital Dr. Abraham Godoy Peña, al realizar desinclusión de diente 3.8 se daña la pared lingual. A la cuarta semana la paciente mantiene dificultad para mover la lengua por pérdida de propiocepción, además se mantenía la parestesia total del lado izquierdo de la lengua, el piso y la encía del mismo lado. Se decide realizar la reconstrucción del NL. Misma técnica quirúrgica. Se realiza el abordaje (Figura 3), se reconoce el conducto submandibular (Figura 4), con el apoyo de microscopía se ubican y liberan ambos cabos del nervio (Figura 5) con el fin de aproximarlo sin tensión para luego realizar la reconstrucción del nervio con microcirugía sobre el epineuro con tres puntos de sutura en $360^{\circ}$,

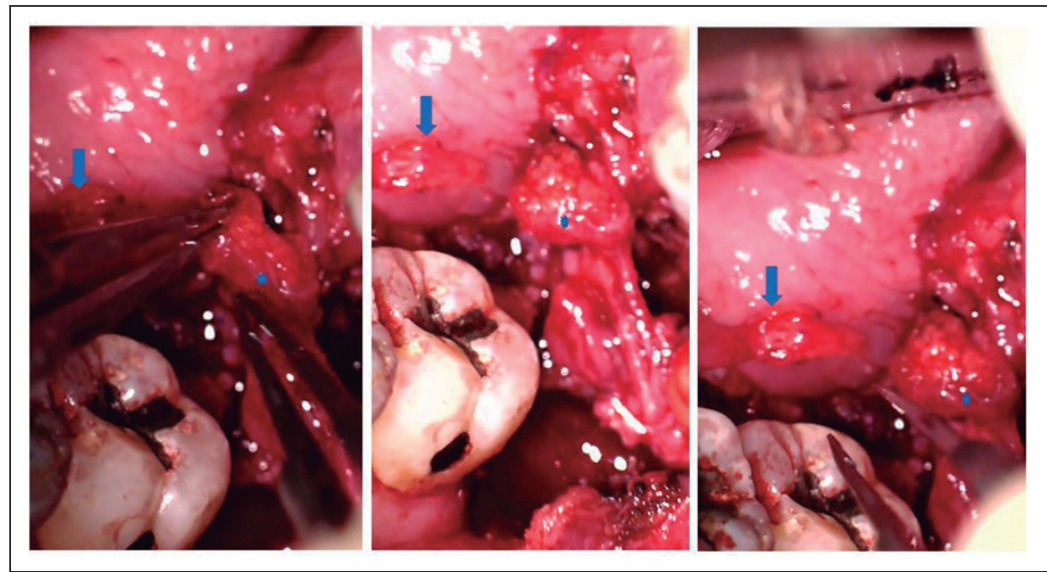

Figura 1. Vista con aumento a través de microscopía: Flecha $\rightarrow$ Muestra el neurofibroma en el cabo proximal del nervio lingual. Asterisco * muestra el cabo distal del nervio lingual.

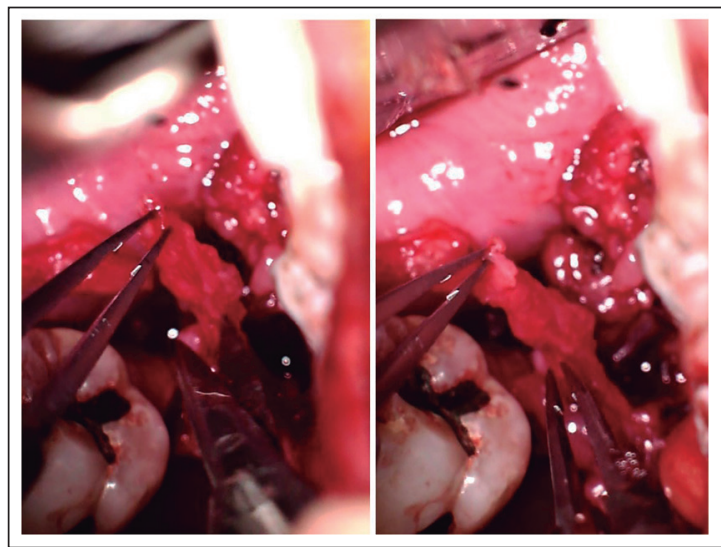

Figura 2. Vista con aumento a través de microscopía. Lado Izquierdo: Flecha $\rightarrow$ muestra el cabo distal del Nervio lingual. Asteris co * Muestra la disección del tejido fibroso y tejido lacerado del cabo proximal. Lado derecho: Flecha $\rightarrow$ muestra el cabo distal del Nervio lingual. Asterisco * muestra la aproximación para medir la tensión del cabo proximal luego de la resección del nervio. 


\section{CASOS CLÍNICOS}

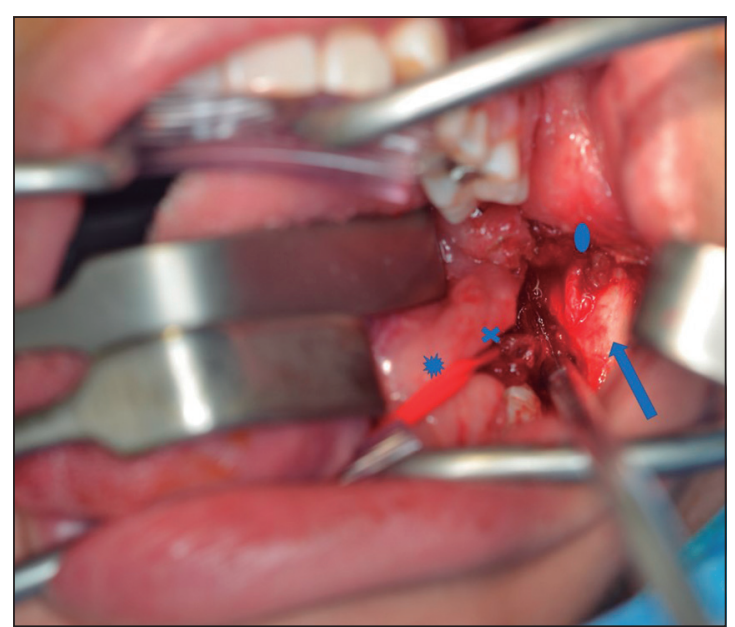

Figura 3. Se muestra el abordaje sobre el piso de boca con la descarga además en vestibular, distal al segundo molar; y las zonas anatómicas relevantes a considerar durante el procedimiento: * piso de boca. $\mathbf{X}$ En profundidad glándula submandibular y el conducto de la glándula. $\rightarrow$ Borde anterior de la rama mandibular. $\mathbf{O}$ En profundidad la língula.

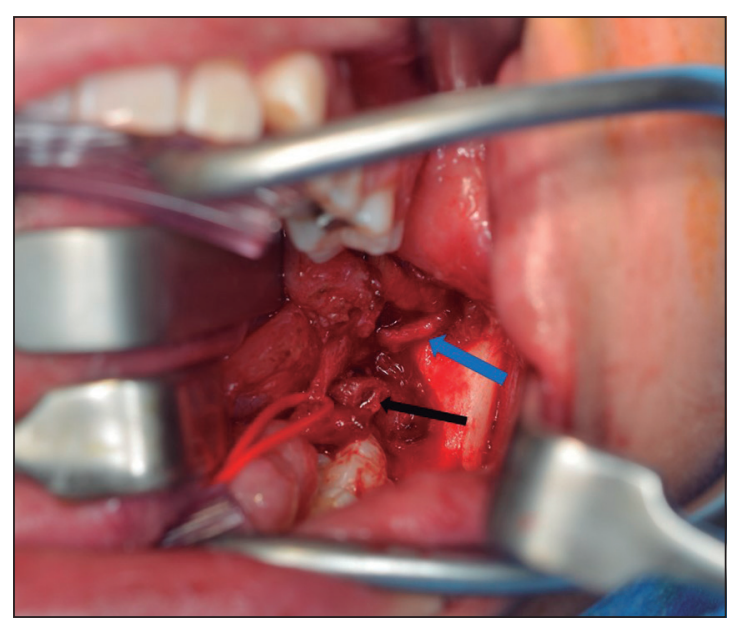

Figura 5. La flecha negra muestra el cabo distal del nervio, mientras que la flecha azul muestra el cabo proximal sobre el borde anterior de la rama.

nylon 8/0 (Figura 6). Al día siguiente la paciente refería que la sensación de compresión en la base de la lengua había desaparecido y percibía el tacto profundo en distintas partes de la lengua (S2). A los 18 meses paciente refiere buena sensibilidad al tacto superficial y profundo (S4) en casi toda la lengua disminuido levemente en piso de boca y FSR +. Al gusto paciente refiere $100 \%$ de recuperación.

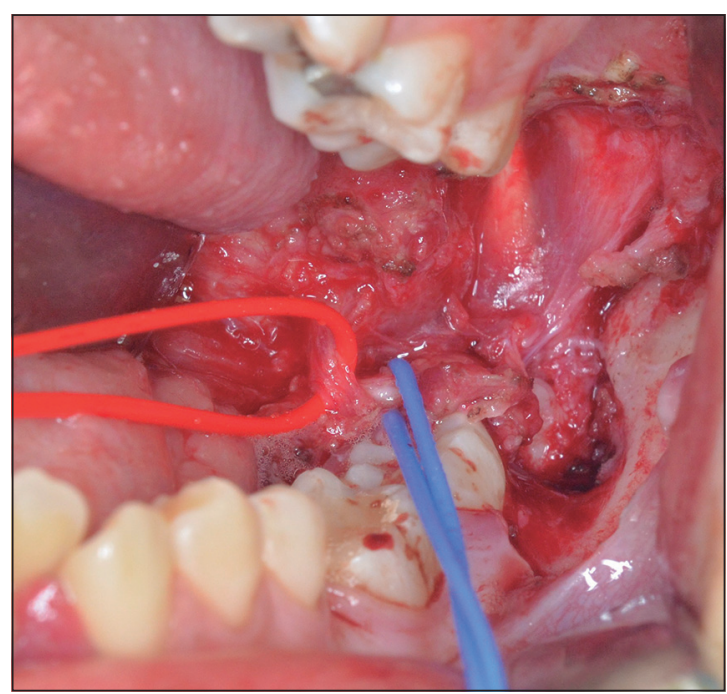

Figura 4. Se muestra el nervio pasando por debajo del conducto de la glándula submandibular (loop). Retractor rojo sostiene al conducto de glándula submandibular izquierda y el azul sostiene al cabo distal del nervio.

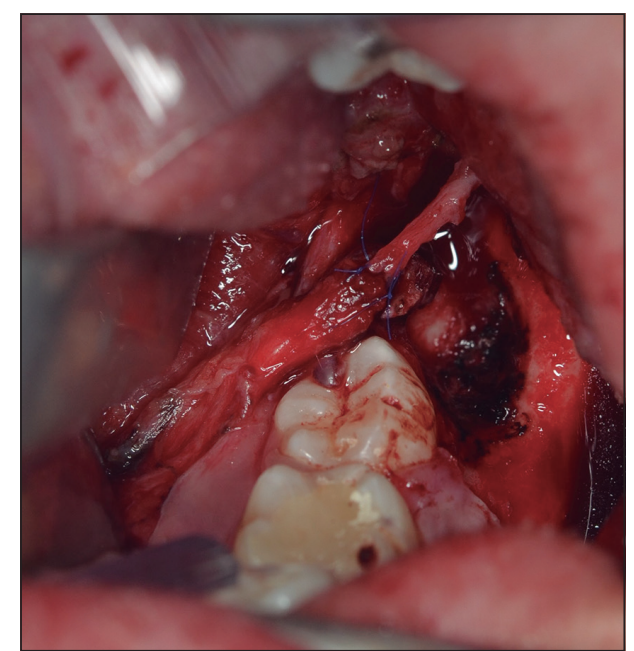

Figura 6. Se muestra la reconstrucción microquirúrgica del nervio lingual en la zona del epineuro en $360^{\circ}$.

\section{Discusión}

El daño del NL está asociado a múltiples procedimientos, entre ellos, resección de tumores o cáncer en la región del cuerpo mandibular, de piso de boca o lengua y osteotomías mandibulares, siendo la desinclusión de terceros molares inferiores la causa más habitual ${ }^{3-5}$. Frecuentemente existe una recuperación 
autónoma del nervio ${ }^{3,4}$, de lo contrario sospechamos una lesión mayor del nervio incluyendo la posibilidad de un neuroma traumático.

La parestesia, pérdida del gusto, dificultad para hablar o deglutir es la principal sintomatología $a^{4,6,12}$. Este tipo de lesión puede llegar a afectar severamente la calidad de vida ${ }^{13}$. Pese a su alta tasa de éxito la reconstrucción microquirúrgica del NL es una técnica que no se desarrolla de forma frecuente en todas partes del mundo. La RNM es el método diagnóstico de elección, además podemos apoyarnos en la evaluación neurofisiológica de conducción nerviosa para obtener mayor información respecto al grado de lesión del NL. En nuestros pacientes no tuvimos acceso a estos exámenes debiendo realizar el diagnóstico clínicamente. El tratamiento microquirúrgico del NL no es frecuente en Chile ni en Sudamérica, siendo éste el primer reporte de casos en nuestro país. No todos los pacientes son candidatos a la reconstrucción del NL; una gran mayoría de ellos logran recuperar su función ${ }^{3,4}$. Si el nervio está seccionado completamente la reconstrucción del nervio debería ser llevada a cabo lo más temprano posible, si es parcial es posible esperar, sin embargo no existe consenso respecto al tratamiento, ni el momento de la cirugía. El año 2000, Scrivani evaluó la capacidad de recuperación del gusto con un éxito sobre el $80 \%{ }^{12}$, Pogrel (tratamiento previo a 3 meses), Susarla y Bhargeri demuestran una alta tasa de éxito en el tratamiento temprano (antes de 6 meses) $(\mathrm{p}<0,05)^{5-7}$. Mientras en casos de alodinia o dolor neuropático, la microcirugía parece ser una buena herramienta ${ }^{11}$. Siguiendo estas recomendaciones, en nuestro estudio ambas pacientes fueron operadas antes de los 3 meses; sin embargo, el año 2019, Nakanishi en su estudio retrospectivo en 70 pacientes no encuentra diferencias significativas a los 24 meses antes o después de 6 meses, solo una recuperación más rápida en el tratamiento temprano ${ }^{10}$; además propone que la evaluación funcional de recuperación sensorial (FSR) es una mejor herramienta de medi- ción que sólo la función sensorial ${ }^{10}$. Miloro presenta una serie de resultados satisfactorios en los cuales propone la reconstrucción indirecta con autoinjerto $\mathrm{o}$ aloinjerto toda vez que la reconstrucción presente tensión o no se logre reconstrucción directa ${ }^{14}$. El avance en técnicas microquirúrgicas, ya sea con microscopia o con lupas de aumento, ha permitido que la reconstrucción nerviosa tenga un alto éxito, sobre el $80 \%$ y $90 \%$ en los diversos estudios ${ }^{7,8,10,13,14}$. Para pacientes con secuelas de más de 2 años se ha propuesto una técnica al nervio contralateral con resultados satisfactorios ${ }^{15}$, sin embargo, no existe gran cantidad de artículos que se refieran a este procedimiento.

\section{Conclusiones}

Parece adecuado esperar no más de 6 meses para la reconstrucción, pero se debe otorgar tiempo suficiente para ver si existe algún grado de recuperación de este nervio. Pese a que aún falta convenir el momento más apropiado y otros aspectos del tratamiento quirúrgico del NL, el éxito demostrado en éste y otros artículos indican que la reconstrucción microquirúrgica debe ser incorporada necesariamente dentro del algoritmo de primera elección en el tratamiento de la lesión del nervio lingual.

\section{Responsabilidades éticas}

Protección de personas y animales. Los autores declaran que para esta investigación no se han realizado experimentos en seres humanos ni en animales.

Confidencialidad de los datos. Los autores declaran que en este artículo no aparecen datos de pacientes.

Conflictos de interés: no hay.

\section{Bibliografía}

1. Kikuta S, Iwanaga J, Kusukawa J, Tubbs RS. An anatomical study of the lingual nerve in the lower third molar area. Anat Cell Biol. 2019;52:140-2. doi: 10.5115/ acb.2019.52.2.140

2. Cheung LK, Leung YY, Chow LK, Wong MC, Chan EK, Fok YH. Incidence of neurosensory deficits and recovery after lower third molar surgery: a prospective clinical study of 4338 cases. Int J Oral Maxillofac Surg. 2010;39:320-6. doi: 10.1016/j.ijom.2009.11.010. Epub 2010 Jan 12. PMID: 20061121.

3. Lata J, Tiwari AK. Incidence of lingual nerve paraesthesia following mandibular third molar surgery. Natl J Maxillofac Surg. 2011;2:137-40. doi: 10.4103/09755950.94467 .
4. Posnick JC, Choi E, Singh N. Lingual nerve injury in association with sagittal ramus osteotomy and bicortical screw fixation: a review of 523 procedures in 262 subjects. Int J Oral Maxillofac Surg. 2016;45:1445-51.

5. Pogrel MA. The results of microneurosurgery of the inferior alveolar and lingual nerve. J Oral Maxillofac Surg. 2002;60:485 
6. Susarla SM, Kaban LB, Donoff RB, Dodson TB. Functional sensory recovery after trigeminal nerve repair. J Oral Maxillofac Surg. 2007;65:605. doi: 10.1016/j.joms.2005.11.115. PMID: 17174765.

7. Bagheri SC, Meyer RA, Khan HA, Kuhmichel A, Steed MB. Retrospective review of microsurgical repair of 222 lingual nerve injuries. J Oral Maxillofac Surg. 2010;68:715-23. doi: 10.1016/j. joms.2009.09.111. Epub 2009 Dec 29. PMID: 20036042.

8. Biglioli F, Lozza A, Colletti G, Allevi F. Objective Assessment of Lingual Nerve Microsurgical Reconstruction, Journal of Craniofacial Surgery 2018;29:e740-e744. doi: 10.1097/ SCS.0000000000004663.

9. Susarla SM, Lam NP, Donoff RB, Kaban LB, Dodson TB. A comparison of patient satisfaction and objective assessment of neurosensory function after trigeminal nerve repair. J Oral Maxillofac Surg. 2005;63:1138-44. doi: 10.1016/j.joms.2005.04.021. PMID: 16094581.

10. Nakanishi T, Yamamoto Y, Tanioka K, Shintani Y, Tojyo I, Fujita S. Effect of duration from lingual nerve injury to undergoing microneurosurgery on improving sensory and taste functions: retrospective study. Maxillofac Plast Reconstr Surg. 2019;41:61. Published 2019 Dec 27. doi: 10.1186/s40902-0190244-y

11. Shintani Y, Ueda M, Tojyo I, Fujita S. Change in allodynia of patients with postlingual nerve repair iatrogenic lingual nerve disorder. Oral Maxillofac Surg. 2020;24:25-9. doi: 10.1007/s10006-019. 00803-z. Epub 2019 Nov 15. PMID: 31729606

12. Scrivani SJ, Moses M, Donoff RB, Kaban LB. Taste perception after lingual nerve repair, Journal of Oral and Maxillofacial Surgery 2000;58:3-5. ISSN 0278-2391. https://doi.org/10.1016/ S0278-2391(00)80003-8.

13. Lam N, Donoff RB, Kaban LB, Dodson TB. Patient satisfaction after trigeminal nerve repair, Oral Surgery, Oral Medicine, Oral Pathology, Oral Radiology, and Endodontology 2003;95:538-43. ISSN 1079-2104. https:// doi.org/10.1067/moe.2003.163.

14. Miloro M, Ruckman P 3rd, Kolokythas A. Lingual Nerve Repair: To Graft or Not to Graft? J Oral Maxillofac Surg. 2015;73:1844-50. doi: 10.1016/j. joms.2015.03.018. Epub 2015 Mar 19. PMID: 25865714.

15. Biglioli F, Allevi F, Colletti G, Lozza A. Cross-tongue procedure: a new treatment for long-standing numbness of the tongue, British Journal of Oral and Maxillofacial Surgery 2015;53: 880-2. ISSN 02664356, https://doi.org/10.1016/j. bjoms.2015.09.001. 